\title{
Evaluation of matrix-assisted laser desorption ionization-time of flight mass spectrometry (MALDI-TOF MS) for identification and clustering of Neisseria gonorrhoeae
}

Anna Carannante ${ }^{1 \dagger}$, Elena De Carolis ${ }^{2+}$, Paola Vacca ${ }^{1}$, Antonietta Vella ${ }^{2}$, Caterina Vocale ${ }^{3}$, Maria Antonia De Francesco ${ }^{4}$, Marco Cusini ${ }^{5}$, Simonetta Del Re ${ }^{6}$, Ivano Dal Conte ${ }^{7}$, Antonio Cristaudo ${ }^{8}$, Patrizia Ober ${ }^{9}$, Maurizio Sanguinetti ${ }^{2}$ and Paola Stefanelli ${ }^{{ }^{*}}$

\begin{abstract}
Background: The sexually transmitted infection gonorrhea remains a public health concern for becoming resistant to drug treatments available. The purpose of this study was to evaluate the usefulness of the matrix-assisted laser desorption ionization-time of flight mass spectrometry (MALDI-TOF MS) to identify and cluster Neisseria gonorrhoeae.

From a current monitoring in Italy, as part of the European Gonococcal Antimicrobial Surveillance Programme (EURO-GASP), 93 gonococci collected from 2007 to 2012 susceptible (44 isolates) and resistant (49 isolates) to cefixime were selected. Minimum Inhibitory Concentration (MIC) values for cefixime was assessed by Etest carried out in agreement with the manufacturer's instructions and interpreted referring to European Committee on Antimicrobial Susceptibility testing (EUCAST) clinical breakpoints criteria. Data obtained by N. gonorrhoeae multiantigen sequence typing (NG-MAST) and the dendrogram based on the concatenation of porB and tbpB genes were evaluated. MALDI-TOF MS, to reconfirm gonorrhea identification, analyzed single colonies from freshly grown isolates and applied directly on a ground-steel MALDI target plate. For the MALDI-TOF dendrogram cluster analysis, MSPs (Main Spectrum Profile) from each isolate were created acquiring 5000 shots from 10 technical replicates obtained from bacteria extraction.

Results: Molecular typing by NG-MAST showed 28 sequence types (STs); G1407 was the predominant accounting for 75 gonococci. All the 93 gonococci, except one, were correctly identified at species level by MALDI-TOF MS and G1407 isolates were divided into two clusters.

Conclusion: MALDI-TOF MS for a real-time detection and cluster analysis of gonorrhea is a promising tool for surveillance purposes. Moreover, additional studies are required to collect more data on the performance of MALDI-TOF MS for gonococci.
\end{abstract}

Keywords: Neisseria gonorrhoeae, MALDI-TOF MS, G1407, Cluster

\footnotetext{
* Correspondence: paola.stefanelli@iss.it

'Equal contributors

'Departmentof Infectious, Parasitic \& Immune-mediated Diseases, Istituto

Superiore di Sanità, Rome, Italy

Full list of author information is available at the end of the article
} 


\section{Background}

Neisseria gonorrhoeae is responsible for causing gonorrhea, one of the most common bacterial sexually transmitted disease worldwide, affecting 106 million people each year [1]. Although significant progress was made in reducing the incidence of the disease, gonorrhea infection is becoming a much more difficult to treat, increasing morbidity and medical cost [2-4]. In recent years, the emergence of multi-drug resistant (MDR) $N$. gonorrhoeae is a serious problem for treatment options [3, 5-7]. In particular, as reported by the European Centre for Disease Prevention and Control (ECDC), the proportion of gonococci with reduced susceptibility to cefixime, was $5.1 \%$ in 2009 and $7.6 \%$ in 2011, respectively [3, 8]. Sporadic cases due to ceftriaxone-resistant isolates have also been described in Europe $[9,10]$.

Surveillance of antimicrobial resistance (AMR) and MDR gonorrhea, promoting susceptibility testing and studying the genetic mechanism of resistance, is a key point for the control strategy and to combat the spread of resistant gonococci [10-14].

In this regard, the rapid diagnosis and typing should be pursued to enhance surveillance capabilities [15-17].

Currently, one of the reference method for gonorrhea typing is the $N$. gonorrhoeae multiantigen sequence typing (NG-MAST). Many reports highlight the NG-MAST highly discriminatory power, reproducibility, speed, low cost and easy to perform; a public database [http://www.ng-mast.net] for analysis and assignment of the allele numbers and Sequence Type (ST) is available, $[15,17]$. Nowadays, the ST1407, the main ST together with all the related STs, defined the Genogroup (G) 1407 [14-16]. Gonococci belonging to ST1407, mainly associated with reduced susceptibility and resistance to third generation cephalosporins, are spreading in Europe [13-16] and worldwide [18]. In 2011, it was noted in 20/21 European countries and the association among ciprofloxacin resistance, decreased susceptibility to cefixime and G1407 has been observed [15]. Moreover, isolates belonging to G1407 with an increased minimum inhibitory concentrations (MICs) to ceftriaxone and azithromycin, have also been reported [15]. Overall, the identification of a specific genogroup may predict patterns of antimicrobial resistant among circulating gonococci [16]. Recently, the identification by the matrix-assisted laser desorption ionization-time of flight mass spectrometry (MALDI-TOF MS) has revolutionized the workflow of microbiology laboratories $[19,20]$, because of its capability to quickly identify bacterial species and yeasts accurately in comparison with conventional methods [21, 22]. Nowadays, MALDI-TOF MS is considered a reliable tool for the detection and typing of bacteria [23-25].
The purpose of this study was to evaluate the usefulness of MALDI-TOF MS to identify and cluster Neisseria gonorrhoeae.

\section{Methods}

N.gonorrhoea isolates, microbiological methods and patients

Primary isolation, identification and collection, following standard microbiological procedures of 93 gonococci, collected from 2007 to 2012, were performed by the Collaborating Laboratories belonging to Universities, Sexual Transmitted Infectious (STI) and DermatologyVenereology (DV) Clinics. Routine diagnostic testing consisted with culture of $N$. gonorrhoeae from clinical samples (i.e. urethral exudate) inoculating directly on Thayer-Martin medium (Oxoid Ltd, Milan, Italy) with $1 \%$ IsoVitalex (Oxoid, Hampshire, United Kingdom) at $37{ }^{\circ} \mathrm{C}$ in a $5 \% \mathrm{CO}_{2}$ atmosphere. Identification was carried out mainly by using API NH system gallery (bioMérieux, Hantverksvägen, Sweden). Isolates were sent to the Istituto Superiore di Sanità (ISS) for storage $-80{ }^{\circ} \mathrm{C}$ and further microbiological investigations.

Antimicrobial susceptibility tests were performed following the European Gonococcal Antimicrobial Surveillance Programme (EURO-GASP), [26]. In particular, antimicrobial susceptibility to cefixime, was assessed by Etest (bioMérieux, Hantverksvägen, Sweden) in agreement with the manufacturer's instructions. MIC values were interpreted referring to European Committee on Antimicrobial Susceptibility testing (EUCAST) clinical breakpoint criteria $(\mathrm{S} \leq 0.125 \mathrm{mg} / \mathrm{L} ; \mathrm{R}>0.125 \mathrm{mg} / \mathrm{L})$, (version 5.0, 2015), [27]. The World Health Organization (WHO) $N$. gonorrhoeae $\mathrm{G}, \mathrm{K}, \mathrm{M}, \mathrm{O}$, and $\mathrm{P}$ control strains were used in each test [28]. The 93 gonococci were selected referring to antimicrobial susceptibiltiy to cefixime; in particular, 44 isolates were susceptible and 49 resistants. No patient identification was available at the ISS. The isolates were collected for surveillance purposes, thus this work was considered for public health practice, as routine in clinical setting, and no ethical approval was required.

\section{NG-MAST analysis}

Chromosomal DNA was extracted using the QIAamp DNA minikit (Qiagen, Hilden, Germany) according to the manufacturer's instructions. For NG-MAST analysis, sequence of $p o r B$ and $t b p B$ genes were performed using primers and amplification parameters, as previously described [29]. The porB and $t b p B$ alleles were assigned at the NG-MAST website (http://www.ng-mast.net), following the interpretative criteria [29]. Closely related STs were clustered using published definitions $[15,16]$. In particular, all STs which shared one allele and showed $>99 \%$ similarity in the other allele $(\leq 5 \mathrm{bp}$ 
difference for $\operatorname{por} B$ and $\leq 4$ bp for $\operatorname{tbp} B$ ), were included in the same genogroup [15].

NG-MAST data were analyzed by Unweighted Pair Group Mean Average (UPGMA) clustering method using MEGA software (version 5.2, http://www.megasoftware.net); the distance matrices were based on a bootstrap test of 500 replicates; the evolutionary distances were computed using the Tajima-Nei method and visualized as a dendrogram.

\section{MALDI-TOF MS and data analysis}

For MALDI-TOF MS identification, single colonies from freshly grown isolates were picked and directly applied in duplicate on a ground-steel MALDI target plate. One microliter of a saturated solution of $\alpha$-cyano-4-hydroxy cinnamic acid, HCCA (Bruker Daltonik, Bremen, Germany) matrix was deposited on the sample tested and allowed to co-crystallize at room temperature.

Spectra useful for identification were recorded in positive linear mode by a Microflex LT mass spectrometer with a laser frequency of $20 \mathrm{~Hz}$; ion source 1 and 2 voltage, $20 \mathrm{kV}$ and $16.7 \mathrm{kV}$ respectively; lens voltage, $8.5 \mathrm{kV}$ After the automatic acquisition spectra were identified by MALDI Biotyper RTC software, database version 3.1 (4613 entries). For instrument calibration bacterial test standard BTS255343 from Bruker Daltonics it has been used. For the evaluation of the identification results we adopted criteria suggested by the manufacturer; briefly, score values below 1.7 indicated a not reliable identification, between 1.7 and 1.99 a probable genus identification and equal or above 2.0 a secure genus identification and probable or highly probable species identification (between $2.0-2.29$ and above 2.3, respectively). For the MALDI-TOF dendrogram, 93 MSP (Main Spectrum Profile) of the $N$. gonorrhoeae isolates were created to acquire at least 20 separate spectra obtained from 240 shots for a total of above 5000 shots from 10 technical replicates from bacteria extraction with formic acid and acetonitrile, as reported by manufacturer [30]. Quality control peaks was performed using flexAnalysis software (Version 3.3); spectra with outlier peaks or anomalies were removed from the set of the isolate; peaks from 3000 to 10,000 Da were selected and checked so that the allowed difference between the smallest and the largest mass was always lower than $500 \mathrm{ppm}$. Software settings for MSP creation were those suggested by the Bruker Biotyper 3.1 Version [31]. Reproducible spectra profiles was assessed by composite correlation index analysis (CCI) as a representative value of around high conformance of the spectra [32] The hierarchical cluster analysis was performed with the integrated statistical tool Matlab 7.1 of the BioTyper 3.0 software package using default correlation function. Basing on the values obtained from the pairwise comparison of different spectra, a dendrogram was generated allowing the visualization of similarities among spectra profiles.

\section{Results}

\section{NG-MAST analysis}

The molecular typing identified 28 STs out of the 93 isolates. Figure 1 shows the STs found among G1407. In particular, ST1407 was found in 57 isolates (61.3\%), with porB 908 and $t b p B 110$ alleles. According to the genogroup definition, and basing on sequence similarity of the porB and $t b p B$ alleles, ST 2212, 4359, 3499, 8096, 4973, 5570, 7050, 4974, 5335 and 8095 were included in G1407 (75 isolates). The remaining 18, showed different STs, as singletons, except for two belonging to the same ST5339. Out of 18 isolates, 6 belonged to 3 different

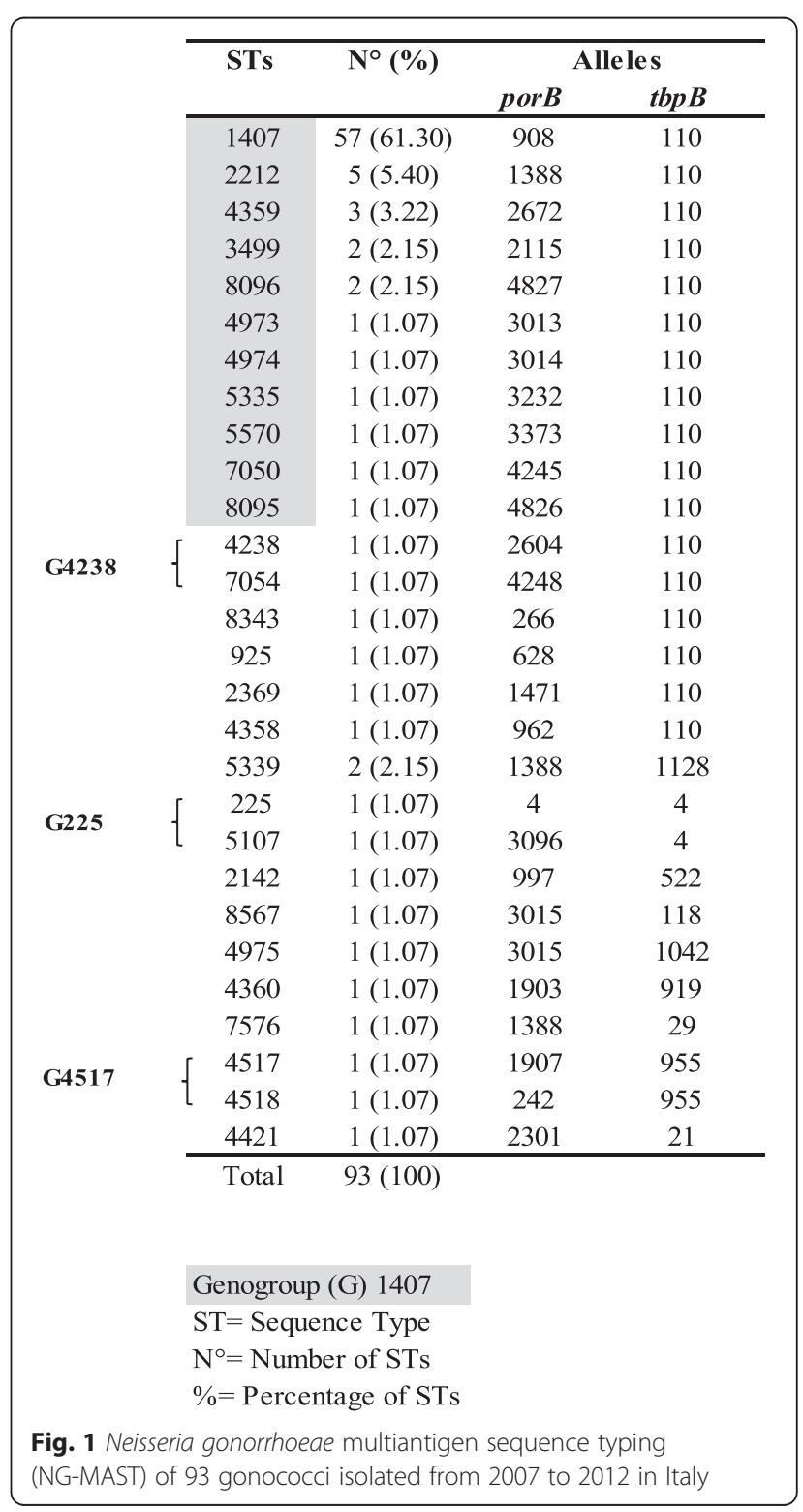


genogroups, according to the definition [15]: G225, G4238, G4517 (Fig. 1).

Figure 2 shows the similarity obtained from $t b p B$ and por $B$ sequences detected in all the isolates.

\section{MALDI-TOF MS analysis}

MALDI-TOF MS allows to correctly identify, at the species level all the 93 isolates, of which $30.1 \%$ with excellent score values (above 2.3); while one isolate was identified at genus level only (with a score of 1.987). The latter was resistant to cefixime and belonging to G1407. One minute per sample was the time estimated from the picking of the colony to obtain the identification results at the species level and 30 cents the cost per sample [21].

As shown in Fig. 3, in relation to their mass signals and intensities, a hierarchic dendrogram clustered the 93 gonococci in two main groups: a large group $(n=77$ isolates) it has been divided into two sub-clusters, the remaining 15 isolates were grouped into a smaller one.
Conversely, 1 single isolate was grouped in a different cluster, according to the arbitrary distance level of 600 (Fig. 3). Dendrograms obtained from MALDI-TOF MS hierarchical cluster analysis and concatenation of $t b p B$ and porB sequences were compared; the G1407 clustered as a single clone by NG-MAST, whilst turn to be divided in two groups by dendrogram obtained with MALDI-TOF MS analysis. In particular, in one cluster have been included isolates belonging to G1407 except for one that had the ST4232 with the tbpB allele 110 present among the G1407 isolates. In the second cluster, 60 out of 77 isolates belonged to G1407. The remaining 17 isolates defined small groups of 2 isolates, i. e. ST225 and ST5107 or ST4517 and ST4518 (Fig. 3).

\section{Discussion}

Gonorrhea is considered the most common bacterial sexually transmitted infection and remains a public health concern due to the spread of antimicrobial resistance $[3,6,33]$. As typing tool, NG-MAST, is routinely

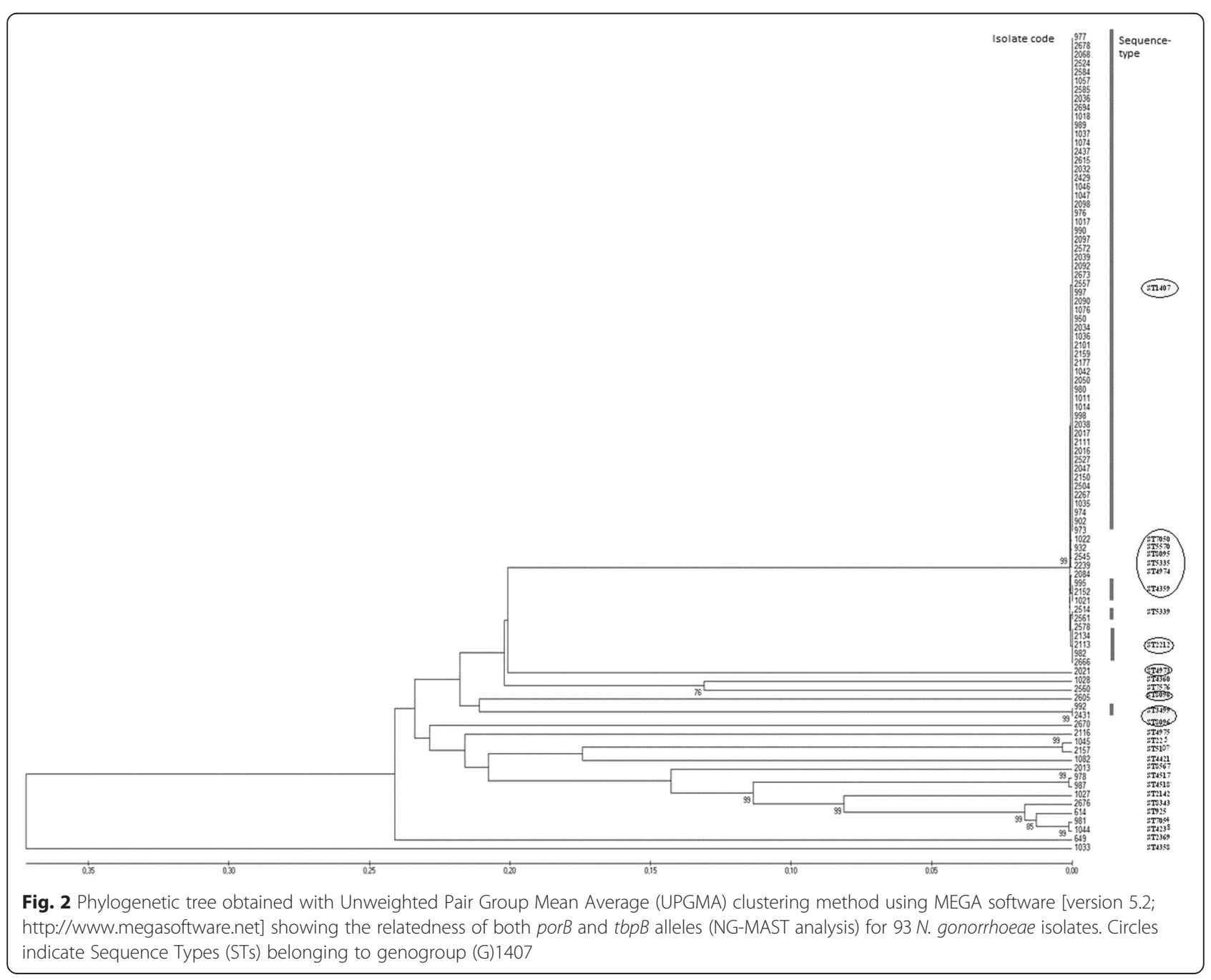




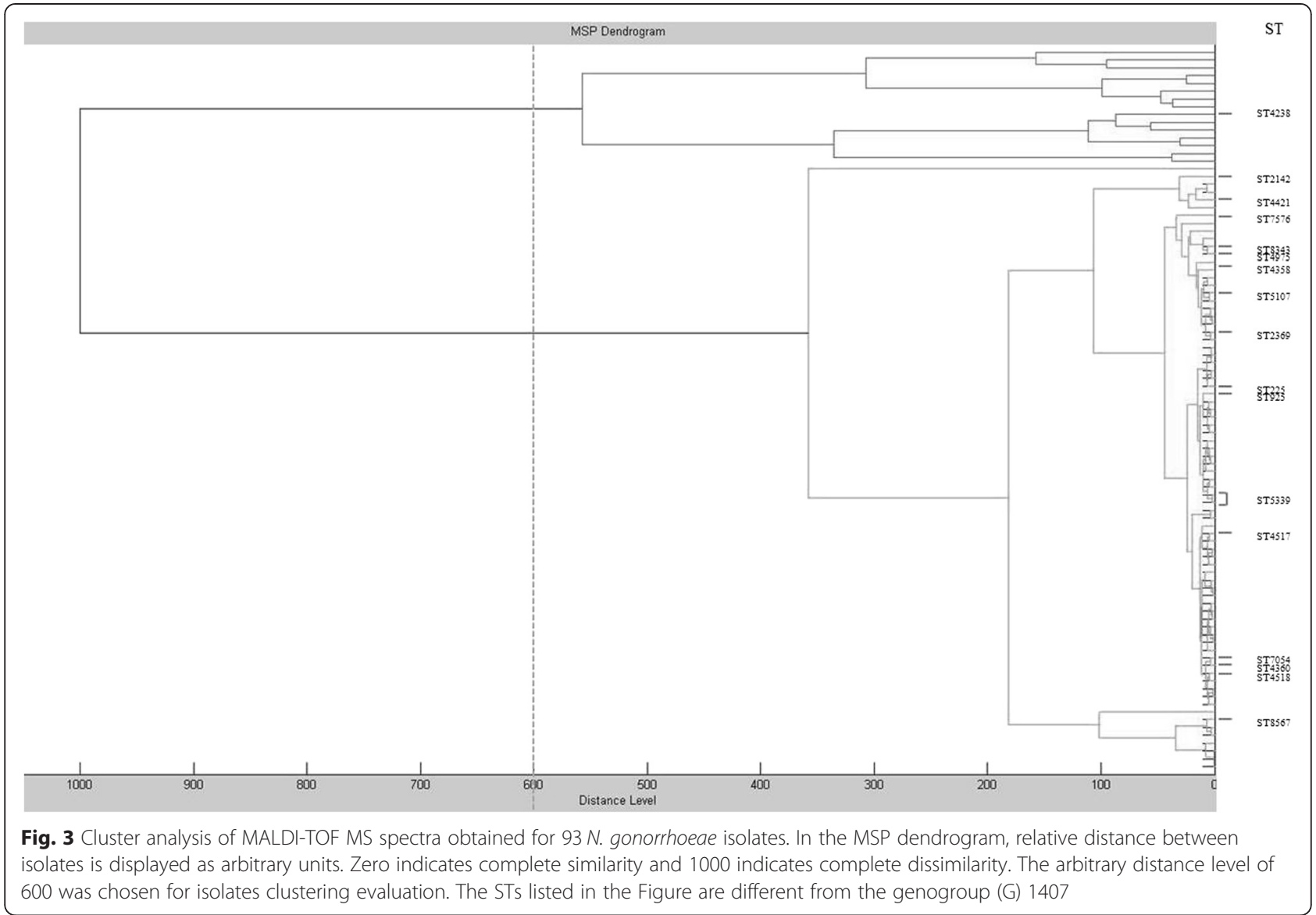

used for the investigation of molecular epidemiology of gonococci as the reference method [14, 16, 34, 35]. Thus, it refers to the sequence variations in two hypervariable loci, $\operatorname{por} B$ and $t b p B$, coding for the gonococcal outer membrane porin and the $\beta$-subunit of the transferrin-binding protein, respectively [15, 29]. Furthermore, it is considered the current method of choice and widely used to identify the route of transmission [15]; several studies have shown a relationship between the NG-MAST and antimicrobial susceptibility profiles [10, 13, 14, 16, 34, 35].

Over the past few years, MALDI-TOF MS has become widely used for the rapid identification and typing of yeast and bacterial species in the clinical microbiology laboratory [23-25]. MALDI-TOF MS identification is based on the differences of whole cell proteins, which are mainly ribosomal proteins, by matching against a database.

In this study we evaluated the ability of MALDI-TOF MS for the rapid identification and cluster of gonococci particularly those belonging to G1407, the genogroup primarly associated with cefixime resistance. This clonal group represented the largest group in our study. Beyond this, gonococci were correctly identified by MALDI-TOF
MS at the species level, except one, at the genus level only, with a score very close to the cut-off of 2.0. Compared to conventional biochemical methods used for the identification, mass spectrometry can be performed in few minutes. Further analysis would be required in order to evaluate the use of MALDI-TOF MS for diagnosis directly on clinical sample.

Moreover, the performance of MALDI-TOF MS for clustering of $93 \mathrm{~N}$. gonorrhoeae isolates, already analysed by NG-MAST, were evaluated as a complement typing method, in particular for screening purposes.

The results showed that all the investigated gonococci were highly related and that those belonging to G1407 were divided into two distinct clusters by MALDI-TOF MS. Although, NG-MAST and MALDI-TOF mass spectrometry rely on quite different approaches, genome and proteome analysis respectively, the results suggest a role for MALDI-TOF MS for at least gonorrhoea identification. Nevertheless, as it is possible to argue, from the comparison between NG-MAST and MALDI-TOFdendrograms, not a reliable use for $N$. gonorrhoeae genotyping is attributable to the latter technique, even if inside the same genogroup, as identified by NG-MAST, the MALDI TOF MS clustered the isolates in more groups. In this context, we 
would like to mention the recent minireview by Spinali et al. [36]. In that article, it is well summarized the need to share guidelines for interpreting the data as already established for other techniques as Pulsed-field gel electrophoresis (PFGE). Furthermore, the potential of MALDI-TOF MS for typing purposes is contradictory from many examples, from E. faecium to L. pneumophila, [36].

Overall, some benefits may be attributed to the use of MALDI-TOF MS: the short time for the identification the low cost, the limited number of colonies needed in comparison with conventional methods.

\section{Conclusion}

In summary the results are promising to consider MALDI-TOF MS for real-time identification of $N$. gonorrhoeae at the species level, with a reduction of time, consumables and skilled personnel compared to conventional methods. Finally, mass spectrometry approach needs to be further evaluated for gonorrhea typing purposes, even if a potential ability to discriminate among isolates belonging to the same genogroup was evinced.

\section{Availability of supporting data}

The matrix obtained from the alignment of $93 N$. gonorrhoeae porB and $t b p B$ genes concatenation has been deposited in TreeBase link to the dataset DOI http://purl.org/ phylo/treebase/phylows/study/TB2:S17893.

\begin{abstract}
Abbreviations
MDR: Multi-drug resistant; ECDC: European Centre for Disease Prevention and Control; AMR: Antimicrobial resistance; NAATs: Nucleic acid amplification tests; NG-MAST: N. gonorrhoeae multiantigen sequence typing; STs: Sequence types; G: Genogroup; MIC: Minimum Inhibitory Concentration; MALDI-TOF MS: Matrix-assisted laser desorption ionization-time of flight mass spectrometry; STI: Sexual Transmitted Infectious; DV: Dermatology-Venereology; EURO-GASP: European Gonococcal Antimicrobial Surveillance Programme; EUCAST: European Committee on Antimicrobial Susceptibility testing; WHO: The World Health Organization; UPGMA: Unweighted Pair Group Mean Average; HCCA: a-cyano-4-hydroxy cinnamic acid; MSP: Main Spectrum Profile; CCI: Composite correlation index analysis; ESCs: Extended-spectrum cephalosporins; PFGE: Pulsed-field gel electrophoresis.
\end{abstract}

\section{Competing interests}

The authors declare that they have no competing interests.

\section{Authors' contributions}

AC performed NG-MAST analysis, completed the antimicrobial susceptibility testing and drafted the manuscript; EDC performed MALDI-TOF MS, analyzed data and contributed to draft the manuscript; PV contributed in the DNA extraction and sequence analysis; AV contributed in the MALDI-TOF MS analysis; CV, MADF, MC, SDR, IDC, AC, PO were in charge in data collection and management of samples; MS critically reviewed the manuscript and the analysis; PS designed the purpose of the study, its coordination, and wrote the manuscript. All the Authors have reviewed and agreed on the content of the final manuscript.

\section{Acknowledgments}

Neisseria gonorrhoeae Italian Study Group: Valeria Ghisetti, R. Milano: Microbiology and Virology Laboratory, Infectious Diseases, Amedeo di Savoia Hospital, Turin; A. Di Carlo, G. Palamara, G. Prignano, A. Latini: San Gallicano
Dermatologic Institute-IRCSS-Rome; L. Scioccati, S. Ramoni: U.O. Dermatology Fondazione Policlinico Mangiagalli Regina Elena, Milan; A. Matteelli: Institute of Infectious and Tropical Diseases, University of Brescia; V. Sambri, MP. Landini, F. Cavrini: Azienda Ospedaliero-Universitaria di Bologna Policlinico S.Orsola-Malpighi U.O. of Microbiology; A. D'Antuono: Azienda OspedalieroUniversitaria di Bologna Policlinico S.Orsola-Malpighi U.O. of Dermatology STDs Center, Bologna ; J. Fiore, Raffaele Antonetti, A. Di Taranto, R. De Nittis: Dept. of Clinical Pathology, Azienda Ospedaliero-Universitaria OORR, Foggia; M. Gaino, R. Predazzer: Microbiology and Virology Laboratory, Santa Chiara Hospital, Trento; F. Urbani: U.O. Dermatology, Santa Chiara Hospital, Trento; M. Busetti, V. Petix, T. Rossi: Institute for Maternal and Child Health - IRCCS "Burlo Garofolo", Trieste; M.A. Latino: Laboratorio di Batteriologia, Ospedale Ostetrico Ginecologico Sant'Anna, Turin; A. Mencacci: Microbiology Section, Dept. of Experimental Medicine and Biochemical Sciences, University of Perugia.

The study was supported by Italian Ministry of Health (grant CCM 2013), the project titled was "Sorveglianza di laboratorio della farmaco resistenza di Neisseria gonorrhoeae come malattia emergente: implementazione della rete di laboratori sul territorio nazionale e caratterizzazione microbiologica dei ceppi farmaco resistenti come richiesto da sorveglianza europea".

\section{Author details}

'Departmentof Infectious, Parasitic \& Immune-mediated Diseases, Istituto Superiore di Sanità, Rome, Italy. ${ }^{2}$ Istituto di Microbiologia, Università Cattolica del Sacro Cuore, Rome, Italy. ${ }^{3}$ Unit of Clinical Microbiology, CRREM Laboratory, St. Orsola-Malpighi, University Hospital, Bologna, Italy. ${ }^{4}$ Dipartimento di Medicina Molecolare e Traslazionale, Sezione di Microbiologia, University of Brescia, Brescia, Italy. ${ }^{5}$ Fondazione IRCCS Ca' Granda Ospedale Maggiore Policlinico, Milan, Italy. ${ }^{6}$ Microbiology and Virology Laboratory, Infectious Diseases, Amedeo di Savoia Hospital, Turin, Italy. ${ }^{7}$ MD, Dip-GUM. STI Clinic, Department of Infectious Diseases, Amedeo di Savoia Hospital, Turin, Italy. ${ }^{8}$ San Gallicano Dermatologic Institute-IRCSS-Rome, Rome, Italy. ${ }^{9}$ Microbiology and Virology Laboratory, Santa Chiara Hospital, Trento, Italy.

Received: 3 February 2015 Accepted: 8 July 2015

Published online: 24 July 2015

\section{References}

1. World Health Organization (WHO). Department of Reproductive Health and Research. Global incidence and prevalence of selected curable sexually transmitted infections - 2008. Geneva, Switzerland: WHO; 2012. http://www.who.int/reproductivehealth/publications/rtis/2008_STI_estimates.pdf.

2. Tapsall JW. Neisseria gonorrhoeae and emerging resistance to extended spectrum cephalosporins. Curr Opin Infect Dis. 2009;22:87-91.

3. Cole MJ, Spiteri G, Chisholm SA, Hoffmann S, Ison CA, Unemo M, et al. Emerging cephalosporin and multidrug-resistant gonorrhea in Europe. Euro Surveill. 2014; 19:pii = 20955. http://www.eurosurveillance.org/NiewArticle.aspx?Articleld=20955.

4. Ohnishi M, Golparian D, Shimuta K, Saika T, Hoshina S, Iwasaku K, et al. Is Neisseria gonorrhoeae initiating a future era of untreatable gonorrhea?: detailed characterization of the first strain with high-level resistance to ceftriaxone. Antimicrob Agents Chemother. 2011;55:3538-45.

5. European Centre for Disease Prevention and Control (ECDC). Surveillance report. Response plan to control and manage the threat to multidrug-resistance gonorrhoea in Europe. Stockholm, 2012. ECDC; 2012. http://www.ecdc.europa.eu/en/publications/Publications/1206-ECDC-MDRgonorrhoea-response-plan.pdf

6. World Health Organization (WHO). Department of Reproductive Health and Research. Global action plan to control the spread and impact of anti-microbial resistance in Neisseria gonorrhoeae. Geneva, Switzerland: WHO; 2012. http://whalibdoc.who.int/publications/2012/9789241503501_eng.pdf.

7. Carannante A, Renna G, Dal Conte I, Ghisetti V, Matteelli A, Prignano G, et al. Changing antimicrobial resistance profiles among Neisseria gonorrhoeae isolates in Italy, 2003 to 2012. Antimicrob Agents Chemother. 2014;58:5871-76.

8. European Centre for Disease Prevention and Control (ECDC). Surveillance report. Gonococcal antimicrobial susceptibility surveillance in Europe, 2011. Stockholm, 2013. ECDC; 2013. http://www.ecdc.europa.eu/en/ publications/publications/gonococcal-antimicrobial-susceptibilitysurveillance-27-mar-2013.pdf. 
9. Camàra J, Serra J, Ayats J, Bastida T, Carnicer-Pont D, Andreu A, et al. Molecular characterization of two high-level ceftriaxone-resistant Neisseria gonorrhoeae isolated detected in Catalonia, Spain. J Antimicrob Chemother. 2012;67:1858-60.

10. Unemo M, Golparian D, Nicholas R, Ohnishi M, Gallay A, Sednaoui P. High-level cefixime and ceftriaxone resistant $N$. gonorrhoeae in France: novel penA mosaic allele in a successful international clone causes treatment failure. Antimicrob Agents Chemother. 2012;56:1273-80.

11. Shimuta K, Unemo M, Nakayama S, Morita-Ishihara T, Dorin M, Kawahata T, et al. Antimicrobial resistance and molecular typing of Neisseria gonorrhoeae isolates in Kyoto and Osaka, Japan, 2010 to 2012: intensified surveillance after identification of the first strain ( $\mathrm{H041}$ ) with high-level ceftriaxone resistance. Antimicrob Agents Chemother. 2013;57:5225-32.

12. Starnino S, Suligoi B, Regine V, Bilek N, Stefanelli P, Neisseria gonorrhoeae Italian Study Group, et al. Phenotypic and genotypic characterization of Neisseria gonorrhoeae in parts of Italy: detection of a multiresistant cluster circulating in a heterosexual network. Clin Microbiol Infect. 2008;14:949-54.

13. Carannante A, Prignano G, Cusini M, Matteelli A, Dal Conte I, Ghisetti V, et al. Cefixime and ceftriaxone susceptibility of Neisseria gonorrhoeae in Italy from 2006 to 2010. Clin Microbiol Infect. 2012;18:558-64.

14. Jeverica S, Golparian D, Matičič M, Potočnik M, Mlakar B, Unemo M. Phenotypic and molecular characterization of Neisseria gonorrhoeae isolates from Slovenia, 2006-12: rise and fall of the multi-drug-resistant NG-MAST genogroup 1407 clone? J Antimicrob Chemother. 2014;69:1517-25.

15. European Centre for Disease Prevention and Control (ECDC). Technical report. Molecular typing of Neisseria gonorrhoeae. Results from a pilot study 2010-2011. Stockholm, 2012. ECDC; 2012. http://www.ecdc.europa.eu/en/ publications/Publications/201211109-Molecular-typing-gonorrhea.pdf.

16. Chisholm SA, Unemo M, Quaye N, Johansson E, Cole MJ, Ison CA, et al. Molecular epidemiological typing within the European Gonococcal Antimicrobial Resistance Surveillance Programme reveals predominance of a multidrug-resistant clone. Euro Surveill. 2013;18:20358-68.

17. Unemo M, Dillon JR. Review and international recommendation of methods for typing Neisseria gonorrhoeae isolates and their implications for improved knowledge of gonococcal epidemiology, treatment, and biology. Clin Microbiol Rev. 2011:24:447-58.

18. Pandori M, Barry PM, Wu A, Ren A, Whittington WL, Liska S, et al. Mosaic penicillin-binding protein 2 in Neisseria gonorrhoeae isolates collected in 2008 in San Francisco, California. Antimicrob Agents Chemother. 2009:53:4032-4.

19. Bizzini A, Greub G. Matrix-assisted laser desorption ionization time-of-flight mass spectrometry, a revolution in clinical microbial identification. Clin Microbiol Infect. 2010;16:1614-9.

20. Wieser A, Schneider L, Jung J, Schubert S. MALDI-TOF MS in microbiological diagnostics-identification of microorganisms and beyond (mini review). Appl Microbiol and Biotechnol. 2012;93:965-74

21. Tan KE, Ellis BC, Lee R, Stamper PD, Zhang SX, Carroll KC. Prospective evaluation of a matrix-assisted laser desorption ionization-time of flight mass spectrometry system in a hospital clinical microbiology laboratory for identification of bacteria and yeasts: a bench-by-bench study for assessing the impact on time to identification and cost-effectiveness. J Clin Microbiol. 2012;50:3301-8

22. Perez KK, Olsen RJ, Musick WL, Cernoch PL, Davis JR, Land GA, et al. Integrating rapid pathogen identification and antimicrobial stewardship significantly decreases hospital costs. Arch Pathol Lab Med. 2013;137:1247-54.

23. Ayyadurai S, Flaudrops C, Raoult D, Drancourt M. Rapid identification and typing of Yersinia pestis and other Yersinia species by matrix-assisted lase desorption/ionization time-of-flight (MALDI-TOF) mass spectrometry. BMC Microbiol. 2010;10:285

24. Zautner AE, Masanta WO, Tareen AM, Weig M, Lugert R, Groß U, et al. Discrimination of multilocus sequence typing-based Campylobacter jejun subgroups by MALDI-TOF mass spectrometry. BMC Microbiol. 2013;13:247.

25. Fournier PE, Drancourt M, Colson P, Rolain JM, La Scola B, Raoult D. Modern clinical microbiology: new challenges and solutions. Nat Rev Microbiol. 2013;11:574-85.

26. Spiteri G, Cole M, Unemo M, Hoffman S, Ison C, Van de Laar M. The European Gonococcal Antimicrobial Surveillance Programme (Euro-GASP)- a sentinel approach in the European Union (EU)/European Economic Area (EEA). Sex Transm Infect. 2013;89:16-8.
27. The European Committee on Antimicrobial Susceptibility Testing (EUCAST) Breakpoints tables for interpretation of MICs and zone diameters, Version 5.0, 2015. Available at: http://www.eucast.org

28. Unemo M, Fasth $\mathrm{O}$, Fredlund $\mathrm{H}$, Limnios A, Tapsall J. Phenotypic and genetic characterization oft he 2008 WHO Neisseria gonorrhoeae reference strain panel intended for global quality assurance and quality control of gonococcal antimicrobial resistance surveillance for public health purposes. J Antimicrob Chemother. 2009;63:1142-51.

29. Martin IM, Ison CA, Aanensen DM, Fenton KA, Spratt BG. Rapid sequence-based identification of gonococcal transmission clusters in a large metropolitan area. J Infect Dis. 2004;189:1497-505.

30. Spanu T, De Carolis E, Fiori B, Sanguinetti M, D'Inzeo T, Fadda G, et al. Evaluation of matrix-assisted laser desorption ionization-time-of-flight mass spectrometry in comparison to rpoB gene sequencing for species identification of bloodstream infection staphylococcal isolates. Clin Microbiol Infect. 2011;17:44-9.

31. Rettinger A, Krupka I, Grünwald K, Dyachenko V, Fingerle V, Konrad R, et al. Leptospira spp. strain identification by MALDI TOF MS is an equivalent too to 165 rRNA gene sequencing and multi locus sequence typing (MLST). BMC Microbiology. 2012;12:185

32. Vella A, De Carolis E, Vaccaro L, Posteraro P, Perlin DS, Kostrzewa M, et al. Rapid antifungal susceptibility testing by matrix-assisted laser desorption ionization time-of-flight mass spectrometry analysis. J Clin Microbiol. 2013:51:2964-69.

33. Bignell C, Unemo M on behalf of the European STI Guidelines Editorial Board. 2012 European guideline on the diagnosis and treatment of gonorrhoea in adults. Int J STD AIDS. 2013;24:85-92. http://www.iusti.org/ regions/Europe/pdf/2012/Gonorrhoea_2012.pdf.

34. Thakur SD, Levett PN, Horsman GB, R Dillon JA. Molecular epidemiology of Neisseria gonorrhoeae isolates from Saskatchewan, Canada: utility of NG-MAST in predicting antimicrobial susceptibility regionally. Sex Transm Infect. 2014;90:297-302

35. Goire N, Lahra MM, Chen M, Donovan B, Fairley CK, Guy R, et al. Molecular approaches to enhance surveillance of gonococcal antimicrobial resistance. Nat Rev Microbiol. 2014;12:223-9.

36. Spinali S, Van Belkum A, Goering RV, Girard V, Welker M, Van Nuenen, et al. Microbial typing by matrix-assisted laser desorption ionization-tima of flight mass spectrometry: do we need guidance for data interpretation? J Clin Microbiol. 2015:53:760-5.

\section{Submit your next manuscript to BioMed Central and take full advantage of:}

- Convenient online submission

- Thorough peer review

- No space constraints or color figure charges

- Immediate publication on acceptance

- Inclusion in PubMed, CAS, Scopus and Google Scholar

- Research which is freely available for redistribution 